\title{
Summary on fruit identification methods: A literature review
}

\author{
Fangyuan Liu ${ }^{1, a}$, Leonid Snetkov², Dimas Lima ${ }^{3}$ \\ ${ }^{1}$ School of Computer Science and Technology, Nanjing Normal University, Jiangsu 210023, China \\ ${ }^{2}$ ITMO University, Kronverksky Prospect 197101, Russian Federation \\ ${ }^{3}$ Department of Electrical Engineering, Federal University of Santa Catarina, Florianópolis, Brazil \\ a2191513453@qq.com
}

Keywords: fruit detection; pattern recognition; classification; computer vision; image processing

\begin{abstract}
Purposes) This paper shows the importance and necessity of intelligent identification technology of fruit detection. (Methods) We enumerate several state-of-the-art methods and illustrate the specific application in the process of recognition, by selecting eleven highly related literature. (Results) On this basis, we make an analysis and comparison on the advantages and disadvantages of each approaches. (Conclusion) This summary can be beneficial to researchers who are interested in fruit identification.
\end{abstract}

\section{Introduction}

With the vigorous development of fruit industry, the use of effective technical methods to classify all kinds of fruits is a general trend [1, 2]. As we all know, manual check is not popular any more, we should apply pattern recognition methods to deal with this problem.

The emergence and development of pattern recognition technology is based on the people who use visual and auditory to identify various information [3, 4]. Pattern recognition is a state-of-the-art technique to process complex information automatically using computer and mathematical theory [5]. For these reasons, the researchers use pattern recognition as an intelligent technology to replace and even expand human daily mental activities. Pattern recognition is used in many domains, such as remote sensing [6, 7], multiple sclerosis detection [8, 9], Alzheimer's disease identification [10, 11], cerebral microbleeding detection [12,13], breast cancer classification [14,15], tea category identification [16, 17], brain image classification [18-20], etc.

The methods covered in this paper are applied on analyzing the category information of the fruit. We focus mainly on fruit detection and compare the accuracy of each approaches.

\section{Methods}

We searched the newly-published literatures related to fruit identification in several important academic databases: Web of Science, Elsevier, Springerlink, IEEE, Engineering Village, etc. Eleven literatures are selected and described below.

Ref. [21] presented a novel method that on the basis of multi-feature fusion to identify five kinds of fruits. The authors take advantage of global histogram, LBP, HOG and GaborLBP for selecting the optimal block. Before that it was proved that the four feature extraction methods when used together have the highest accuracy. Finally, the result achieves $81.35 \%$ using LibSVM.

Ref. [22] put forward a novel approach to classify fruits. Firstly, they use the split-and-Merge algorithm to complete the process of image segmentation. Then, to extract fruit futures on the combination of color histogram, texture and shape. The most important is that they construct three different SVMs: (i) WTA-SVM; (ii) MWV-SVM; (iii) DAG-SVM and choose three different kernels: (i) LIN; (ii) HPOL; (iii) GRB. In the test phase, not only the accuracy is discussed and the time computation of each classifier is also compared.

Ref. [23] designed an approach which focuses on extracting color and texture features. There are two descriptors, CLD and EHD, to extract features after the fruit image segmentation. And then the 
author takes advantage of SVM to accomplish the classification task. Their best result is $100 \%$ in the experiment of distinguishing fruit category.

Ref. [24]created a hybrid feature set and introduced FCSABC-FNN as a classifier. The feature set consist of fruit color, texture and shape and these conditions are achieved by color histogram, Unser's texture feature vector and mathematical morphology measures respectively.

Ref. [25] introduced a robust system based on "SESH + RF" to recognize fruits. SESH represents a combination of several feature descriptors, using SCD to extract color features, using EHD to evaluate texture information, using $\mathrm{Hu} 7$ and $\mathrm{SH}$ to select shape features. The performance of the category method is achieved $99.36 \%$, so the prospect is encouraging.

Ref. [26] applied SIFT to extract features according to the fruits' appearance and using Random Forest (RF) as the classifier to determine the classes of fruits. The author compares RF with the following classifiers: (i) KNN; (ii) SVM. "SIFT + RF" achieves the best performance. However, the datasets of three kinds of fruits are unbalanced.

Ref. [27] proposed an effective methodology for fruit classification system. The highlight in this paper is that the author combines BBO and FNN to recognize eighteen fruit classes. In order to verity the superiority of BBO-FNN, which is compared with five existing methods. The performance of the proposed approach achieves $89.11 \%$.

Ref. [28] provided two novel approaches: (i) WE + PCA + BBO-FNN; (ii)WE + PCA + FCSABC-FNN to classify fruits. BBO and FCSABC are both used to train FNN and show better performance when compare with GA, PSO and ABC. The accuracy of the two methods is the same after testing in this literature.

Ref. [29] used co-occurrence matrix and RBPNN with a statistical algorithm on detecting fruit surface defects. In this process, calculating three co-occurrence matrices to extract effective features and using RBPNN to categorize the defect areas. The accuracy of this experiment is $97.25 \%$ that shows the effectiveness of the method strongly.

Ref. [30] developed a new idea to transform each image into a one-dimension signal for solving problems caused by data-richness. The approach includes four primarily steps: (1) to acquire hyperspectral images that can evaluate the damage areas; (2) to complete hyperspectral images segmentation through a quick threshold way; (3) to construct the appropriate hyper spectrogram; (4) to use iPLS-DA to classify the extent of fruit bruises.

Ref. [31] developed a fuzzy incremental learning algorithm (FILA). Their proposed algorithm are smart computer vision systems. It can grade mangoes in four different types, determined based on value and distances of nearest markets.

\section{Results and Discussions}

Table 1 comparison of accuracy of several methods

\begin{tabular}{|c|c|c|}
\hline Method & Disadvantage & Accuracy \\
\hline Color+HOG+LBP+GaborLBP +LibSVM [21] & The classifier needs to be strengthened. & $81.35 \%$ \\
\hline MWV-SVM + GRB [22] & The number of significant features is insufficient. & $88.2 \%$ \\
\hline Color and texture feature + SVM [23] & This work only extracts two vital features. & $100 \%$ \\
\hline Hybrid feature set and FCSABC-FNN [24] & The prediction is unstable. & $89.1 \%$ \\
\hline SESH + RF [25] & The computational complexity is high. & $99.36 \%$ \\
\hline SIFT + RF [26] & The datasets are unbalanced in fruit category. & $96.97 \%$ \\
\hline $\begin{array}{c}\mathrm{CH}+\text { Unser's texture measure + morophology + } \\
\text { BBO-FNN [27] }\end{array}$ & $\begin{array}{l}\text { The performance of feature extraction is lower } \\
\text { than other methods. }\end{array}$ & $89.11 \%$ \\
\hline $\begin{array}{c}\text { WE + PCA +BBO-FNN } \\
\text { WE + PCA + FCSABC-FNN [28] }\end{array}$ & $\begin{array}{l}\text { There are other classifiers superior to FNN and } \\
\text { have better performance. }\end{array}$ & $89.5 \%$ \\
\hline Co-occurrence and RBFNN [29] & $\begin{array}{l}\text { Classification speed is slower than other } \\
\text { algorithms. }\end{array}$ & $97.25 \%$ \\
\hline Hyperspectrogram + iPLS-DA [30] & Classification is time-consuming. & $94.04 \%$ \\
\hline FILA [31] & sensitive to the maturity and quality & $87 \%$ \\
\hline
\end{tabular}


In general, we discuss the accuracy and weakness of each method that is mentioned above. The results are listed in Table 1 . We gave the methodology, disadvantage, and accuracy of each method. Note that all the accuracies were recorded from the literatures themselves.

The support vector machine (SVM) is a powerful tool in traditional classifiers. The researchers are suggested to test advanced SVM classifiers, such as fuzzy SVM [32-35], generalized eigenvalue proximal SVM [36, 37], twin SVM [38, 39], etc.

In the future, we suggest researchers to apply deep learning method on fruit identification. Some typical deep learning techniques, such as convolutional neural network [40], sparse autoencoder, and deep belief network, have been successfully applied in computer vision. The researchers can learn from their applications, and test the feasibility of deep learning in fruit classification.

\section{Conclusion}

This study describes several pattern recognition methods from a series of articles for identify fruit information. The most effective approach can be found from this paper and the researchers are able to develop better ideas on this basis. Furthermore, pattern recognition methods are applied in other fields has a bright future.

\section{Acknowledgement}

The paper is financially supported by Fundamental Research Funds for the Central Universities (LGYB201604), Open fund for Jiangsu Key Laboratory of Advanced Manufacturing Technology (HGAMTL1601), Open fund of Key Laboratory of Guangxi High Schools Complex System and Computational Intelligence (2016CSCI01).

\section{References}

[1] Pardo-Mates, N., A. Vera, et al., Characterization, classification and authentication of fruit-based extracts by means of HPLC-UV chromatographic fingerprints, polyphenolic profiles and chemometric methods. Food Chemistry, 2017. 221: pp. 29-38

[2] Khan, A., J.P. Li, et al. Vision Based Classification of Fresh Fruits Using Fuzzy Logic. in 10th Indiacom - 3rd International Conference on Computing for Sustainable Global Development. 2016. New Delhi, INDIA: IETE. pp. 3932-3936

[3] Wu, L.N., Pattern Recognition via PCNN and Tsallis Entropy. Sensors, 2008. 8(11): pp. 7518-7529

[4] Dong, Z. and G. Ji, Effect of spider-web-plot in MR brain image classification. Pattern Recognition Letters, 2015. 62: pp. 14-16

[5] O'Brien, R.J., J.M. Fontana, et al., A pattern recognition system based on acoustic signals for fault detection on composite materials. European Journal Of Mechanics a-Solids, 2017. 64: pp. 1-10

[6] Wu, L. and G. Wei, A New Classifier for Polarimetric SAR Images. Progress in Electromagnetics Research, 2009. 94: pp. 83-104

[7] Naggaz, N. and G. Wei, Remote-sensing Image Classification Based on an Improved Probabilistic Neural Network. Sensors, 2009. 9(9): pp. 7516-7539

[8] Zhou, X.-X., Comparison of machine learning methods for stationary wavelet entropy-based multiple sclerosis detection: decision tree, k-nearest neighbors, and support vector machine. Simulation, 2016. 92(9): pp. 861-871

[9] Zhan, T.M. and Y. Chen, Multiple Sclerosis Detection Based on Biorthogonal Wavelet Transform, RBF Kernel Principal Component Analysis, and Logistic Regression. IEEE Access, 2016. 4: pp. 7567-7576 
[10] Du, S., Alzheimer's Disease Detection by Pseudo Zernike Moment and Linear Regression Classification. CNS \& Neurological Disorders - Drug Targets, 2017. 16(1): pp. 11-15

[11]Wang, S.-H. Single slice based detection for Alzheimer's disease via wavelet entropy and multilayer perceptron trained by biogeography-based optimization. Multimedia Tools and Applications, 2016, DOI: 10.1007/s11042-016-4222-4 (Online).

[12] Chen, Y. Voxelwise detection of cerebral microbleed in CADASIL patients by leaky rectified linear unit and early stopping: A class-imbalanced susceptibility-weighted imaging data study. Multimedia Tools and Applications, 2016, DOI: 10.1007/s11042-017-4383-9 (Online).

[13]Chen, H. Seven-layer deep neural network based on sparse autoencoder for voxelwise detection of cerebral microbleed. Multimedia Tools and Applications, 2017, DOI: 10.1007/s11042-017-4554-8 (Online).

[14] Rao, R.V., A. Liu, et al., Abnormal Breast Detection in Mammogram Images by Feed-forward Neural Network trained by Jaya Algorithm. Fundamenta Informaticae, 2017. 151(1-4): pp. 191-211

[15] Liu, G., Computer-aided diagnosis of abnormal breasts in mammogram images by weighted-type fractional Fourier transform. Advances in Mechanical Engineering, 2016. 8(2), Article ID: 11

[16] Cattani, C. and R. Rao, Tea Category Identification Using a Novel Fractional Fourier Entropy and Jaya Algorithm. Entropy, 2016. 18(3), Article ID: 77

[17] Liu, A., Tea Category Identification using Computer Vision and Generalized Eigenvalue Proximal SVM. Fundamenta Informaticae, 2017. 151(1-4): pp. 325-339

[18] Sun, P., Preliminary research on abnormal brain detection by wavelet-energy and quantum-behaved PSO. Technology and Health Care, 2016. 24(s2): pp. S641-S649

[19] Feng, C., Feed-forward neural network optimized by hybridization of PSO and ABC for abnormal brain detection. International Journal of Imaging Systems and Technology, 2015. 25(2): pp. 153-164

[20] Yang, G., Automated classification of brain images using wavelet-energy and biogeography-based optimization. Multimedia Tools and Applications, 2016. 75(23): pp. 15601-15617

[21] Kuang, H.L., L.L.H. Chan, et al., Multi-Class Fruit Detection Based on Multiple Color Channels. Proceedings of 2015 International Conference on Wavelet Analysis and Pattern Recognition (Icwapr), 2015: pp. 1-7

[22] Zhang, Y.D. and L.N. Wu, Classification of Fruits Using Computer Vision and a Multiclass Support Vector Machine. Sensors, 2012. 12(9): pp. 12489-12505

[23] Rachmawati, E., I. Supriana, et al., Multiclass Fruit Classification of RGB-D Images Using Color and Texture Feature. Intelligence in the Era of Big Data, Icsiit 2015, 2015. 516: pp. 257-268

[24] Zhang, Y.D., S.H. Wang, et al., Fruit classification using computer vision and feedforward neural network. Journal of Food Engineering, 2014. 143: pp. 167-177

[25] Jiang, L.X., A. Koch, et al., Multi-class Fruit Classification using RGB-D Data for Indoor Robots. 2013 IEEE International Conference on Robotics and Biomimetics (Robio), 2013: pp. 587-592

[26] Zawbaa, H.M., M. Hazman, et al., Automatic fruit classification using random forest algorithm. 2014 14th International Conference on Hybrid Intelligent Systems (His), 2014: pp. 164-168

[27] Zhang, Y.D., P. Phillips, et al., Fruit classification by biogeography-based optimization and feedforward neural network. Expert Systems, 2016. 33(3): pp. 239-253 
[28] Wang, S.H., Y.D. Zhang, et al., Fruit Classification by Wavelet-Entropy and Feedforward Neural Network Trained by Fitness-Scaled Chaotic ABC and Biogeography-Based Optimization. Entropy, 2015. 17(8): pp. 5711-5728

[29] Capizzi, G., G. Lo Sciuto, et al., Automatic Classification of Fruit Defects based on Co-Occurrence Matrix and Neural Networks. Proceedings of the 2015 Federated Conference on Computer Science and Information Systems, 2015. 5: pp. 861-867

[30] Ferrari, C., G. Foca, et al., Fast exploration and classification of large hyperspectral image datasets for early bruise detection on apples. Chemometrics and Intelligent Laboratory Systems, 2015. 146: pp. 108-119

[31] Nandi, C.S., B. Tudu, et al., A Machine Vision Technique for Grading of Harvested Mangoes Based on Maturity and Quality. Ieee Sensors Journal, 2016. 16(16): pp. 6387-6396

[32] Yang, J., Identification of green, Oolong and black teas in China via wavelet packet entropy and fuzzy support vector machine. Entropy, 2015. 17(10): pp. 6663-6682

[33] Liu, G., Pathological brain detection in MRI scanning by wavelet packet Tsallis entropy and fuzzy support vector machine. SpringerPlus, 2015. 4(1), Article ID: 716

[34] Lu, H.M., Facial Emotion Recognition Based on Biorthogonal Wavelet Entropy, Fuzzy Support Vector Machine, and Stratified Cross Validation. IEEE Access, 2016. 4: pp. 8375-8385

[35] Li, Y., Detection of Dendritic Spines using Wavelet Packet Entropy and Fuzzy Support Vector Machine. CNS \& Neurological Disorders - Drug Targets, 2017. 16(2): pp. 116-121

[36] Yang, J., Preclinical diagnosis of magnetic resonance (MR) brain images via discrete wavelet packet transform with Tsallis entropy and generalized eigenvalue proximal support vector machine (GEPSVM). Entropy, 2015. 17(4): pp. 1795-1813

[37] Liu, A., Magnetic resonance brain image classification via stationary wavelet transform and generalized eigenvalue proximal support vector machine. Journal of Medical Imaging and Health Informatics, 2015. 5(7): pp. 1395-1403

[38] Chen, M., Morphological analysis of dendrites and spines by hybridization of ridge detection with twin support vector machine. PeerJ, 2016. 4, Article ID: e2207

[39] Yang, M., Dual-Tree Complex Wavelet Transform and Twin Support Vector Machine for Pathological Brain Detection. Applied Sciences, 2016. 6(6), Article ID: 169

[40] Orlando, J.I., E. Prokofyeva, et al. Convolutional Neural Network Transfer for Automated Glaucoma Identification. in 12th International Symposium on Medical Information Processing And Analysis. 2017. Tandil, ARGENTINA: Spie-Int Soc Optical Engineering 